\title{
Numerical Analysis Viscoelasticity Properties COMPOSITE OF WOOD
}

\author{
Murco Obucina $^{\mathrm{a}}$, Ejub Dzaferovic ${ }^{\mathrm{a}}$, Enil Gondzic ${ }^{\mathrm{b}}$ \\ ${ }^{a}$ Faculty of Mechanical Engineering, University of Sarajevo, V. setaliste 9, Sarajevo 71000, Bosnia and Herzegovina \\ ${ }^{b}$ Company Standard, Bosanski put 103, Sarajevo 71000, Bosnia and Herzegovina
}

\begin{abstract}
Research is the viscoelasticity properties composite of wood made by gluing beech peeled veneer and at constant load, and two constant humidity. Based on the experimental results obtained by the method of the least squares rheological parameters have been determined as well as their differences in relation depending on the moisture content of samples. Has been developed and applied numerical model based on the finite volume method analysis of viscoelasticity properties composite of wood. Starting with the continuum concept we presented and broadened the application of the finite volume method for solving of the hygro-viscoelasticity behaviour of the composite of wood. Have been ignored of anisotropy in wood, and observation of the sample as homogenous, non-layered material, and also neglect of glue. We developed the constitutive relation including the impact of moisture, expressed through relaxation functions and given in integral form. The calculation results are compared with results of the experiment
\end{abstract}

Keywords: LVL composite; gluing; creep; viscoelasticity properties; finite volume method; numerical model
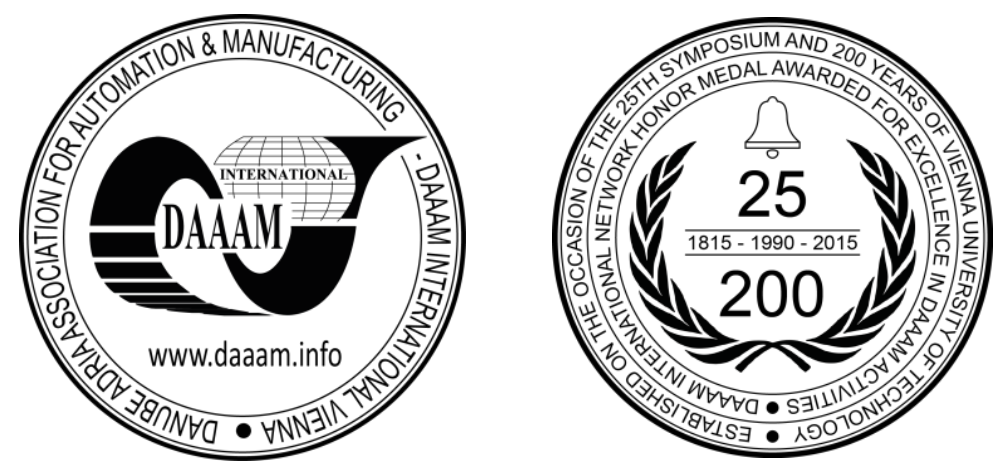

This Publication has to be referred as: Obucina, M[urco]; Dzaferovic, E[jub] \& Gondzic, E[nil] (2016). Numerical Analysis Viscoelasticity Properties Composite of Wood, Proceedings of the 26th DAAAM International Symposium, pp.0182-0188, B. Katalinic (Ed.), Published by DAAAM International, ISBN 978-3-902734-07-5, ISSN 1726-9679, Vienna, Austria DOI:10.2507/26th.daaam.proceedings.025 


\section{Introduction}

For the analysis of stability of a wood construction product exposed to loading is important to know what kind of correlation exists between deformation $(\varepsilon)$ and stress $(\sigma)$. It was believed for a long time that linear correlation between stress and deformation was sufficient for product construction design This was confirmed by a number of researches conducted $[3,4,5,7]$.

By summing up the results of those researches it may be considered that wood behaves as an elastic material at sufficiently law values of loading, moisture, temperature and long loading periods. Wood behaves as linear viscoelasticity material at medium loading values and it has considerable non-linear properties at high loading values.

By knowing viscoelasticity materials characteristics, good agreement of estimated results and real behavior of wood products has been reached. Viscoelasticity wood behavior is very complex and current theories describe it as unsteady relations between forces, deformations, temperature and moisture content. The two important characteristics of viscoelasticity behavior over time are the creep at constant stress and stress relaxation at constant deformation.

One of the ways to describe viscoelastic behavior is by showing correlation between deformation and stress in integral form that is through hereditary integral. Boltzman principle of superposition is used to determine the correlation between deformation and stresses of linear viscoelasticity materials at intermittent stress or deformation loading. Creep resulting from different stress loading values is equal to the sum of all incremental deformations [10].

Ormarson, and. Dahlblom (2013) [8] presents a finite element implementation of a beam element with the aim of studying how wooden composites behave during both mechanical and environmental load action. The beam element is exposed to both axial and lateral deformation. The material model employed concerns the elastic, shrinkage, mechano-sorption and visco-elastic behavior of the wood material. It is used here to simulate the behavior of several simply-supported and continuous composite beams subjected to both mechanical and environmental loading to illustrate the advantages this can provide. The results indicate clearly both the inhomogeneity of the material and the variable moisture action occurring to have had a significant effect on the stress distribution within the cross-section of the products that were studied. Klasnja and Kopitovic (1992) [6] studied juncture strength of three-layer beech board glued with phenol - formaldehyde glue at temperature $155 \square \mathrm{C}$ and $170 \square \mathrm{C}$ and gluing time 11 and 15 minutes. They found out that increased temperature and longer time considerably influenced on glue joint strength.

Use of LVL parts is spreading and in the most cases these products are exposed to constant influence of environment variation (humidity and temperature changes) and those are the factors that have the greatest influence on the mechanical properties of the products and their lifetime, which is often measured in civil engineering by decades.

The wood parts produced by HF gluing are often met in practice. The research has shown that HF glued parts have different deformations compared with the hot glued parts made by conventional hot gluing [9]. Above all, this fact is explained by differences in material warming, moisture content gradient, temperature and gluing time.

In this paper are examples composite of wood obtained by various technological processes gluing subjected to the test of creep under constant load, temperature and two humidity. High (94\%) and low relative humidity (33\%).

Has been elaborated and applied the appropriate numerical algorithm for solving viscoelastic deformation of wood and wood products based on the finite volume $(\mathrm{FV})$ calculation results are compared with the results of the experiment.

\section{Materials and methods}

Pealed beach veneer (Fagus Sylvatica $\mathrm{L}$ ) of the $1^{\text {st }}$ degree of quality, mostly with radial cut, size $550 \times 550 \times 2.1$ $\mathrm{mm}$ and with $5.5 \%$ average moisture content was used for the construction composite of wood type laminated veneer lumber (LVL). Veneers were without errors and were classified according to volume mass.

Melamine-urea-formaldehyde glue E-1 quality was used. Glue was spread over veneer manually by roller in quantity of $180 \mathrm{~g} / \mathrm{m}^{2}$. The specific pressure of $1.8 \mathrm{MPa}$ was applied for gluing. HF generator was used for $\mathrm{HF}$ gluing. Working frequency of the generator was $4.75-6.3 \mathrm{MHz}$, nominal power $6-18 \mathrm{~kW}$ generating $800 \mathrm{~V} / \mathrm{cm}$ strong electrical field between electrodes.

The boards made by conventional hot gluing were marked as $\mathrm{K}$ and the boards made by $\mathrm{HF}$ gluing were marked as HF. After standard conditioning $\left(\mathrm{T}=20^{\circ} \mathrm{C}\right.$ and $\left.\varphi=65 \%\right)$ specimens were cut out of each LVL board for determining relevant mechanical characteristics, as well as 15 specimens, $40 \mathrm{~mm}$ of width, for creep compliance test.

Creep test was carried out on a device with four-wheel loading, where it is possible to perform creep test for 5 elements simultaneously (Figure 1). The device was placed in a chamber where relative humidity was maintained at a constant level (94\%) by axial fans forcing the air to flow over a container with saturated solution of water and $\mathrm{K}_{2} \mathrm{SO}_{4}$. Moisture content level was maintained at $33 \%$ by $\mathrm{MgCl}_{2} \cdot 6 \mathrm{H}_{2} \mathrm{O}$. 


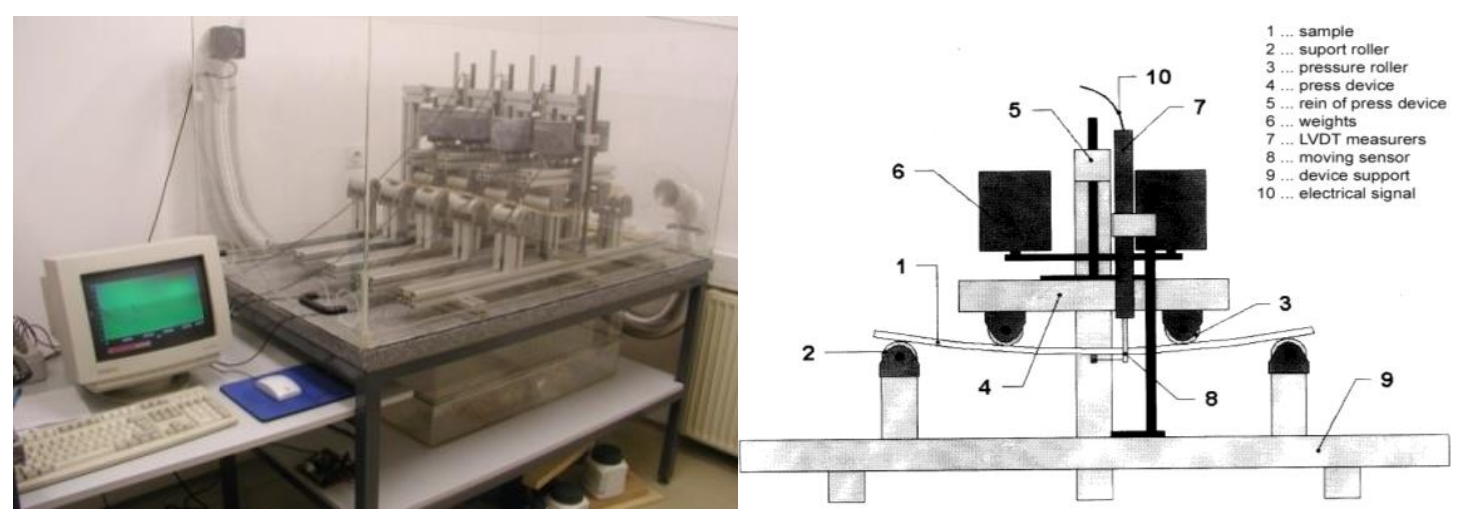

Fig. 1. Device for creep test of LVL specimens

The room temperature was $22,5^{\circ} \mathrm{C}$. Constant loading level was applied by weights (Figure 1, position 6). The mass of the weights together with press device was35,94 kg, which is $17,5 \%$ of the maximum loading (sample fracture). The Figure 1. shows the principle of the load application. Electronic provider LVDT (linear variable differential transformer) with accuracy $\pm 0.0025 \mathrm{~mm}$ was used for deflection measuring. By means of an electronic computer card A/D the processor records data in the first 10 seconds at the rate of $5 \mathrm{data} / \mathrm{measurements}$ per second, then one data/measurement per 2 seconds over the next $600 \mathrm{~s}$ and then one data/measurement per $600 \mathrm{~s}$. Cycle duration for different moisture contents was determined on the basis of experience obtained by preliminary experiments. Prior to this procedure the specimens were first conditioned in the normal environmental conditions $\left(\mathrm{T}=20{ }^{\circ} \mathrm{C} ; \varphi=65 \%\right)$. The creep test lasted 21 days for both environmental conditions. Creep in LVL was observed both on 15 specimen cut out of the boards glued by hot gluing procedure and on 15 specimen cut out of the boards glued by HF procedure.

For the given loading situation, it is considered for viscoelasticity materials that modulus of elasticity (E) is equivalent to relaxation modulus. For moderate loading values (compared to bending strength) and for the linear viscoelastic material behaviour the creep compliance is equivalent to the reciprocal value of elastic modulus.

$J(t)=\frac{1}{E(t)}$

Equation for deflection is now defined as follows:

$\Delta U(t)=\frac{23}{648} \frac{F \cdot l^{3}}{I} \cdot J(t)$

$\Delta U(t)$ - linear viscoelastic deflection $(\mathrm{m})$

$F$-force $(\mathrm{N})$;

$l$ - reference length $(\mathrm{m})$

$I=\frac{b h^{3}}{12}-$ moment of inertia of the beam's cross section $\left(\mathrm{m}^{4}\right)$

$E$-modulus of elasticity $(\mathrm{MPa})$

Parameters of the three-parameter and four-parameter models, which describe viscoelasticity behaviour of LVL elements, may be determined by application of the method of least squares.

\section{Experimental data / Results}

The results obtained by analysis of the experimental data-parameters of the models of elements made both by conventional hot $(\mathrm{K})$ and HF gluing are, respectively, shown in Table 1., while experimental curves and curves represented by corresponding models are shown in Figure 2.

\begin{tabular}{|c|c|c|c|c|c|c|}
\hline Environmental conditions & $\begin{array}{c}\text { Gluing } \\
\text { procedure }\end{array}$ & Model & $\mathrm{E}_{1}[\mathrm{MPa}]$ & $\mathrm{E}_{2}[\mathrm{MPa}]$ & $\begin{array}{c}\mu_{1}[\mathrm{MPa} \\
\text { days }]\end{array}$ & $\begin{array}{c}\mu_{2}[\mathrm{MPa} \\
\text { days }]\end{array}$ \\
\hline \multirow{4}{*}{$\begin{array}{l}\text { Humid } \\
\varphi=94 \%\end{array}$} & \multirow{2}{*}{ Convection } & 3-parametar & $1,2854 \mathrm{e}+04$ & $7,0034 \mathrm{e}+03$ & $3,4506 \mathrm{e}+04$ & \\
\hline & & 4-parametar & $1,4403 e+04$ & $9,3247 \mathrm{e}+03$ & $4,2177 \mathrm{e}+05$ & $2,5296 \mathrm{e}+04$ \\
\hline & \multirow{2}{*}{$\mathrm{HF}$} & 3-parametar & $1,3129 \mathrm{e}+04$ & $9,0014 \mathrm{e}+03$ & $4,0271 \mathrm{e}+04$ & \\
\hline & & 4-parametar & $1,4432 \mathrm{e}+04$ & $1,1604 \mathrm{e}+04$ & $5,6128 \mathrm{e}+05$ & $2,8943 e+04$ \\
\hline \multirow{4}{*}{$\begin{array}{c}\text { Dry } \\
\varphi=33 \%\end{array}$} & \multirow{2}{*}{ Convection } & 3-parametar & $1,7027 \mathrm{e}+04$ & $4,8512 \mathrm{e}+04$ & $1,2361 \mathrm{e}+05$ & \\
\hline & & 4-parametar & $1,7659 \mathrm{e}+04$ & $6,0329 \mathrm{e}+04$ & $2,3820 \mathrm{e}+06$ & $5,0022 \mathrm{e}+04$ \\
\hline & \multirow{2}{*}{$\mathrm{HF}$} & 3-parametar & $1,8036 \mathrm{e}+04$ & $5,5953 \mathrm{e}+04$ & $1,0615 \mathrm{e}+05$ & \\
\hline & & 4-parametar & $1,8566 \mathrm{e}+04$ & $6,6262 \mathrm{e}+04$ & $3,1559 \mathrm{e}+06$ & $4,7371 \mathrm{e}+04$ \\
\hline
\end{tabular}

Table 1. Rheological parameters for deflection at constant loading 

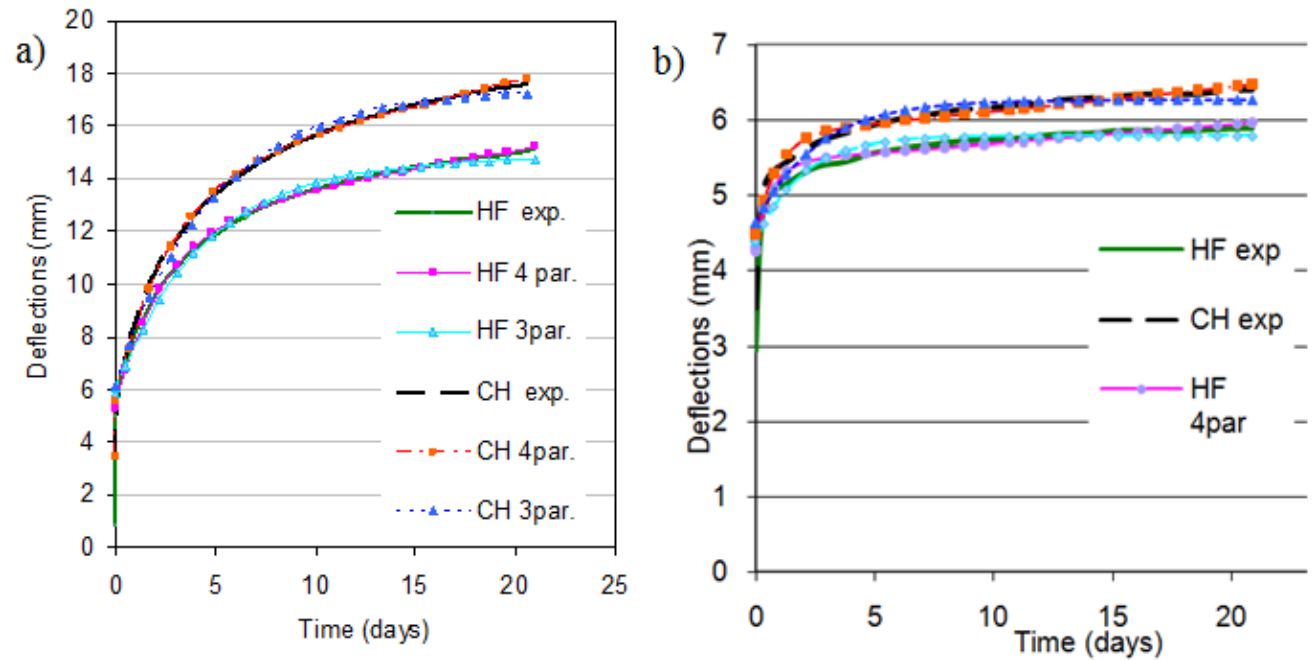

Fig. 2. Measured and calculated creep values of LVL specimens a) humid $\varphi=94 \%$ b) dry $\varphi=33 \%$

The analysis of the creep line and parameters of the applied rheological models showed the effect of moisture changes and gluing procedure on creep in LVL elements. Relative air humidity and humidity content in LVL elements, subjected to constant loading, have a dominant effect on the creep.

After the initial elastic deformation in LVL elements, subjected to loading and high moisture content ( $\varphi=94 \%)$, deformation that was created increased with time. After 21 days a deformation created was two-and-a-half times the size of the deformation in the elements that were exposed to moisture content of $\varphi=33 \%$ (Figure 2).

This increase of deformation can be explained by changes of elastic modulus due to increase of moisture content in LVL elements. Increase of moisture content, in the hygroscopic range, caused decrease in elastic modulus and increase in the deformation, while a long exposure to high moisture decreases the ability of micro-fibrils to resist deformation and physical interaction with matrix material [1].

A type of technological gluing procedure, due to different duration of the applied pressure, affects creep rate in laminated elements. Laminated elements glued by HF gluing procedure showed better rheological properties (lower creep rate), that is they have higher rheological parameters in the applied viscoelasticity three-parameter and fourparameter models, respectively.

\section{Numerical modeling}

The equations that describe the correlation between stress $(\sigma)$ and deformation $(\varepsilon)$ of a viscoelastic solid body are complex and complicated for analytical calculations. Those analytical calculations are mainly related to specific geometrical cases and boundary conditions and as such they are of no practical use. However, with application of numerical methods and computers it is possible to set up a mathematical model and get its numerical solution for a number of practical engineering problems. There are, in principle, two approaches for analysis of both moisture motion and correlation between stress and deformation in solid body. According to the first approach the solid body is treated as a composition of molecules and macroscopic phenomena are described as a consequence of molecular activity and are calculated by application of the laws of mechanics and law of probability (statistical approach). The second approach ignores the effects that occur at the level of micro-mechanism processes whereby the material is treated as continuum. The concept of continuum is based on a premise that its values such as: density, motion, temperature, humidity, etc, may be expressed (in mathematical sense) as continual constitutive functions of time and space.

Viscoelasticity behaviour of wood caused by humidity and temperature changes may be described by basic equations of continuum mechanics. Along with constitutive relations, and initial and boundary conditions they form a closed system of partial differential equations which represents a mathematical model of viscoelasticity behaviour of wood and wood based products.

$\frac{\partial}{\partial t} \int_{V} \rho \frac{\partial(\partial \mathbf{u})}{\partial t} d V=\int_{S} \delta \boldsymbol{\sigma} \cdot \mathbf{n} d S+\int_{V} \rho \boldsymbol{d}_{b} d V$

The equations are valid for both a body as a whole and every part of volume $\mathrm{V}$ encompassed by surface $\mathrm{S}$, with an external unit vector of a line perpendicular to the surface $\mathrm{S}$; whereby $\mathrm{u}$ is a increment vector of displacement, $\delta \sigma$ is increment stress tensor, $\delta f_{b}$ is resultant of the volume increment forces affecting the body, $\rho$ density.

In isotropic viscoelastic materials, the relation between strain history and stress is: 
$\boldsymbol{\sigma}(t)=\int_{0}^{t} 2 \mu(t-\tau) \frac{\delta \boldsymbol{\varepsilon}(\tau)}{\delta \tau} \mathrm{d} \tau+\mathbf{I} \int_{0}^{t} \lambda(t-\tau) \operatorname{tr}\left(\frac{\delta \boldsymbol{\varepsilon}(\tau)}{\delta \tau}\right) \mathrm{d} \tau$

or in an incremental form of stress $\delta \sigma(\mathrm{t})$ due to an increment of strain $\delta \varepsilon(\tau)$ at time $\tau$ is given for $\mathrm{t} \geq \tau$ as:

$$
\delta \boldsymbol{\sigma}(t)=2 \mu(t-\tau) \delta \boldsymbol{\varepsilon}(\tau)+\lambda(t-\tau) \operatorname{tr}(\delta \boldsymbol{\varepsilon}(\tau)) \mathbf{I}
$$

where

$$
\delta \boldsymbol{\sigma}(t), \quad \delta \boldsymbol{\varepsilon}(\tau)=1 / 2\left[\operatorname{grad} \delta \mathbf{u}(\tau)+(\operatorname{grad} \delta \mathbf{u}(\tau))^{\mathrm{T}}\right], \quad \operatorname{tr}(\delta \boldsymbol{\varepsilon}(\tau))=\operatorname{div} \delta \mathbf{u} \text { and } \delta \mathbf{u}
$$

are stress, strain and displacement increments, respectively, and I is the identity tensor. Thus, the solution of the viscoelastic solid body problem reduces to the solution of the following vector equation:

$\frac{\partial}{\partial t} \int_{V} \rho \frac{\partial(\partial \mathbf{u})}{\partial t} d V=\int_{S}\left\{\mu(t-\tau)\left[\operatorname{grad} \delta \mathbf{u}+(\operatorname{grad} \delta \mathbf{u})^{\mathrm{T}}\right]+[\lambda(t-\tau) \operatorname{div} \delta \mathbf{u}] \mathbf{I}\right\} \cdot \mathbf{n} \mathrm{d} S+\int_{V} \delta \mathbf{f}_{b} \mathrm{~d} V$

with the displacement increment vector $\delta \mathrm{u}(\tau)$ as unknown.

This is a small strain formulation in which no distinction is made between deformed and undeformed configurations. There are two independent viscoelastic functions which can have different time dependence. As in the case of elasticity, we may consider engineering functions rather than Lame ones. The viscoelastic time dependent functions are Young's modulus E(t), and Poisson's ratio $v(\mathrm{t})$. Having calculated displacement increments at time $\mathrm{t}$, stress increments at the same time are obtained from Eq. (9), and the total displacements are updated in the current time step:

$$
\mathbf{u}^{m}=\mathbf{u}^{m-1}+\delta \mathbf{u}^{m}
$$

where $m$ is the time-step counter. Calculated displacement increments are stored in time for every computational points, enabling the calculation of stress increments (Eq. (5)) and total stresses (Eq. (4)) at any time t. The total stresses at time $\mathrm{t}$ are updated in the current time step using Eq. (4). The solution is then advanced into the next time step, where the whole procedure is repeated. Since the problem is of an elliptic nature, the boundary conditions have to be specified at all boundaries of the solution domain. They can be either of Dirichlet type (displacement boundary conditions) or of Von Neumann type (traction boundary conditions). In many practical situations the problem exhibits some kind of symmetry in which case only one part of the body may be taken as the solution domain and the symmetry boundary conditions are applied at the interface. In this section the finite volume discretisation of the integral equation (6) is briefly outlined; a more detailed description is available in Reference 6. FV technique is employed to approximate Eq. (6) by a system of algebraic equations, with displacement increment vector components as unknown. The time domain is subdivided into a number of arbitrary time steps. The solution domain is divided into a number of contiguous control volumes (CVs) or cells. Here, only simple geometries are considered and therefore orthogonal meshes are employed. Cells with four faces are used for 2D geometries. A computational node is placed at the centre of each CV and a boundary node, required for the specification of boundary conditions, is placed at the centre of each boundary cell-face. All dependent variables and material properties are stored in the computational nodes.

Then Eq. (6) is written for each control volume as follows:

$$
\frac{\partial}{\partial t} \int_{V} \rho \frac{\partial(\partial \mathbf{u})}{\partial t} d V=\sum_{k} \int_{S_{K}}\left\{\mu\left[\operatorname{grad} \delta \mathbf{u}+(\operatorname{grad} \delta \mathbf{u})^{\mathrm{T}}\right]+(\lambda \operatorname{div} \delta \mathbf{u}) \mathbf{I}\right\} \cdot \mathbf{n} \mathrm{d} S+\int_{V} \delta \mathbf{f}_{b} \mathrm{~d} V
$$

where the summation is over the cell-faces bounding the $\mathrm{CV}, \mu$ and $\lambda$ are functions of $\mathrm{t}-\tau$. In order to solve this equation, a coordinate system must be chosen, integrals have to be approximated and distributions of dependent variables and physical properties in space and time have to be assumed. Components of vectors and tensors related to a global Cartesian system are preferred, as they lead to a strong conservation form of the equilibrium equation. Both surface and volume integrals are approximated employing the mid-point rule. The linear spatial variation of dependent variables is assumed in approximating cell-face values and cell-face gradients. A harmonic interpolation is employed to obtain the cell-face values of mechanical properties. An iterative segregated procedure with decupling is applied. Equations for the Cartesian components of the displacement increment $\delta \mathrm{u}$ are linearized and temporary decoupled by assuming that coefficients and source terms are known. These systems are then solved using conjugate gradient method with preconditioning

The following premises were adopted in the numerical calculation of viscoelastic creep in LVL elements: LVL elements were treated as isotropic material and the glue film is considered as negligible. The calculation is done for 
numerical network of $1600 \mathrm{CV}$ and a plane voltage condition was adopted Calculations of creep in LVL elements were carried out for the four-parameter model with parameter values for the elements glued by conventional HF procedure as well as for two constant values of relative humidity of air, (94\%) i (33\%), respectively. The results of numerical calculation of creep were verified and compared with the results obtained in experiments (Figure 3).

Numerical model based on finite volumes method enables solving of viscoelasticity problems. The difference between the lines obtained by an experiment and those obtained by numerical method of CV is evident from the diagram (Figure 3). The difference between the lines obtained by experiment and those obtained by numerical CV method for conventional gluing procedure is around $11 \%$ after the period of 21 days.

For the experiments where HF gluing procedure was applied the difference was around 7\%. That is a maximum difference between the experimental and numerical results of dislocation. This difference was caused the fact that anisotropy in wood was considered as negligible, and because the procedure was performed with the premise that LVL elements were homogenous non-multilayer material.

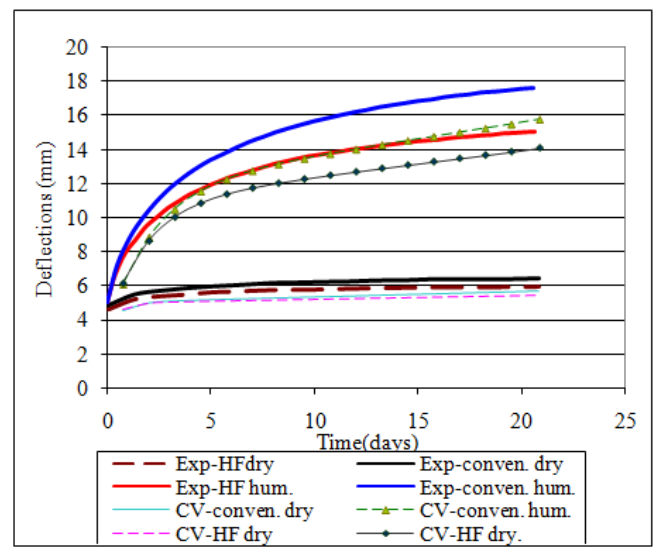

Fig. 3. Creep values measured and obtained by CV method in LVL elements

In the numerical calculations, made some simplifications (neglected the influence of glue, wood is viewed as a homogeneous and isotropic material), which resulted in a particular calculation errors. The numerical model is based on a method FV is a good basis for further research and improvements to the model approach had what a more realistic state of the considered problem.

Recommendations for improving the created mathematical model related to the fact that in future research to develop the mathematical model and numerical algorithm that will consistently to interpret orthotropic properties of wood composites including anisotropic properties of wood and adhesive joint in analyzing the rheological properties.

\section{Conclusion}

The analysis of creep curves and parameters of applied rheological model was established that technological procedure of gluing influence the creep wood composites. Laminated elements glued by HF gluing procedure show better rheological properties (lower creep compliance), that is higher rheological parameters in the applied viscoelastic three-parameter and four-parameter models. The applied model, based on the method of finite volumes, may be used for analysis of viscoelasticity deformations and stresses in multilayer wood products.

Numerical calculation of viscoelastic deformation in LVL elements, with certain simplifications, created difference between the results obtained by the calculations and those obtained by experiments. This difference was caused the fact that anisotropy in wood was considered as negligible, and because the procedure was performed with the premise that LVL elements were homogenous non-multilayer material.

\section{References}

[1] Boyd , J.D., An anatomical explanation for visco-elastic and mechano-sorptive creep in wood and effects loading rate on strength, New

perspectives in wood anatomy, Martinus Nijhoff/ D.W. Junk Publishers, The Hague, Boston, London(1982) 171-222.

[2] Demirdzic, I., Dzaferovic, E., Ivankovic, A., Finite Volume Approach to Thermoviscoelasticity. Numerical Heat Transfer, Part B.47 (2005) 213-237.

[3] Eder, M., Arnould, O., Dunlop, J. W. C., Hornatowska, J., Salmen, L. Experimental micromechanical characterisation of wood cell walls, Wood Sci Technol (2013) 47:163-182

[4] Hanhijarvi, A., Hunt, D., Experimental indication of interaction between viscoelastic and mechano-sorptive creep, Wood Science and Technology 32,(1998), 57-70. 
[5] Hearmon, R. F. S., Paton, J. M. , Moisture Content Changes and Creep of Wood, Forest Products Journal, 14 (1964) 357-359.

[6] Klasnja, B., Kopitovic, S., Lignin-Phenol-Formaldehyde resins as adhesives in the production of plywood, Holz als Roh- und Werkstoff, 50, (1992)7-8: 282-285.

[7] Nakai, T., Grossman,P. U. A., Deflection of wood under intermittent loading. Part I: Fortnightly cycles, Wood Science Technology, 17 (1983) $55-67$.

[8] Ormarson, S. Dahlblom, O., Finite element modelling of moisture related and visco-elastic deformations in inhomogeneous timber beams. Engineering Structures, Volume 49, April 2013, Pages 182-189.

[9] Resnik, J., Tesovnik, F., Thickness loss when gluing veneer sheets into boards in the hot press or by high frequency, Holz als Roh - und Werkstoff,53 (1995) 113-115.

[10] Srpčič , S.,Srpčič , J., Saje, M.in Turk, G. Mechanical analysis of glulam beams exposed to changing humidity. Wood Science and Technology 43 (2009) 1-2: 9-22. 